\title{
Separation of oil-water mixtures using humic acids-coated magnetic nanoparticles
}

Dzhusupkalieva R. ${ }^{1}$, Kydralieva $\mathrm{K}^{2}$

${ }^{1}$ Zhangir Khan West Kazakhstan Agrarian and Technical University, Uralsk, Kazakhstan

${ }^{2}$ Moscow Aviation Institute, Moscow, Russia

Keywords: oil recovery, magnetic nanoparticles, humic acids, composites

doi: 10.36291/HIT.2019.dzhusupkalieva.111

In this study, magnetic nanoparticles (NPs) coated by humic acids (HA) was used to separate a reference VD2 oil from oil-water mixture. Iron oxide NPs are attractive for their inherent low toxicity and magnetism, which allows easy separation of NPs from fluids. Iron oxide NPs were synthesized using a hydrothermal method in air atmosphere. Ferric chloride and ferrous chloride along with HA were used as precursors, and ammonium hydroxide was used as a precipitation agent. AFM images for NPs before and after oil absorption were performed. DLS and zeta potential measurement were performed on a Malvern Nanosizer instrument. Samples were characterized by FTIR spectrometer (FT-IR200, ThermoNicolet). XRD analysis was performed on DRON-UM-2. Oil samples were prepared by mixing crude oil with pure distilled water in vial via sonication $(40 \mathrm{kHz}$, ambient conditions) for 10-60 minutes. Following the preparation of an oil-water mixture, different amounts of NPS were added to the oil-water and mixed for 5-10 min via sonication to obtain optimum oil:NPs ratio. The NPs concentration for the oil experiment was in the range 4-70 ppm. Then, NPs were separated with Nd magnet $(0.3 \mathrm{Tc})$ for specific periods of time. The remaining oil-water solution in each vial was collected for measurement by UV-vis (Shimadzu UV-2600) and fluorescence spectroscopy (Solar CM2203).

The XRD spectrum of the synthesized NPs showed that magnetite $\left(\mathrm{Fe}_{3} \mathrm{O}_{4}\right)$ is the dominant phase of NPs, although the presence of some maghemite cannot be discounted. The average crystal size of 18 and $11 \mathrm{~nm}$ for $\mathrm{Fe}_{3} \mathrm{O}_{4}$ and $\mathrm{Fe}_{3} \mathrm{O}_{4} / \mathrm{HA}$ respectively.

DLS plot for $\mathrm{Fe}_{3} \mathrm{O}_{4}$ and $\mathrm{Fe}_{3} \mathrm{O}_{4} / \mathrm{HA}$ NPs before oil separation experiment had one single peak and the z-average was 140 and $230 \mathrm{~nm}$ respectively, while after oil experiment z-average increases to 310 and $520 \mathrm{~nm}$. Increases in the size of NPs indicate that oil is adsorbed by the NPs and shows the presence of a second layer on the surface of NPs.

To quantify the oil removal, the fluorescence spectrum at excitation wavelength of $337 \mathrm{~nm}$ was recorded. Fluorescence spectroscopy results showed excellent oil removal from oil-water mixture in the pure water in experiments with different concentrations of NPs after 30 minutes of separation. By increasing the NPs concentration from 4 to $40 \mathrm{ppm}$, the oil removal percentage increased from 42 to near $74 \%$. Fluorescence intensity decreased indicating greater removal of aromatic hydrocarbons. These results are in agreement with UV-vis data. Increasing of NPs concentration to $70 \mathrm{ppm}$ lead to NPs aggregation, reducing surface area and absorption capacity. It resulted in oil removal decreasing.

Fluorescence results in the experiments with effect of separation time showed that by increasing the separation time from 10 minutes to 60 minutes, oil removal increased from 32 to near $100 \%$.

In the presence of humic substances, reduction in NPs aggregation and disaggregation has been observed. Moreover, in case of $\mathrm{Fe}_{3} \mathrm{O}_{4} / \mathrm{HA}$ NPs the percentage of the oil removal was higher than for bare $\mathrm{Fe}_{3} \mathrm{O}_{4}$.

Future experiments with simulation of environmental conditions (salinity, $\mathrm{pH}$, ions, natural organic matter, oil grade and concentration) need to be developed. 